\title{
A Survey of Labor and Delivery Practices in New York City during the COVID-19 Pandemic
}

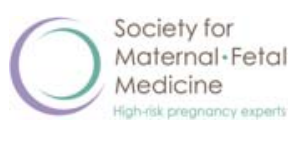

Juan A. Peña, MD, MPH ${ }^{10}$ Angela T. Bianco, MD ${ }^{1}$ Lynn L. Simpson, MD, MSc ${ }^{2}$

Peter S. Bernstein, MD, MPH ${ }^{3}$ Ashley S. Roman, MD, MPH ${ }^{4}$ Dena Goffman, MD $^{2}$

William E. Schweizer, MD, MPH ${ }^{4}$ Jessica Overbey, $\mathrm{DrPH}^{5} \quad$ Joanne L. Stone, MD, MS ${ }^{1}$

${ }^{1}$ Division of Maternal Fetal Medicine, Department of Obstetrics, Gynecology, and Reproductive Science, Icahn School of Medicine at Mount Sinai, New York, New York

2 Division of Maternal Fetal Medicine, Department of Obstetrics and Gynecology, Columbia University Medical Center, New YorkPresbyterian Hospital, New York, New York

${ }^{3}$ Division of Maternal Fetal Medicine, Department of Obstetrics and Gynecology and Women's Health, Albert Einstein College of Medicine/Montefiore Medical Center, Bronx, New York

\begin{abstract}
Address for correspondence Juan A. Peña, MD, MPH, Department of Obstetrics, Gynecology, and Reproductive Sciences, Icahn School of Medicine at Mount Sinai, One Gustave L. Levy Place, New York,

NY 10029 (e-mail: juan.pena@mssm.edu).

${ }^{4}$ Division of Maternal Fetal Medicine, Department of Obstetrics and Gynecology, New York University Langone Health, New York, New York ${ }^{5}$ Department of Population Health Science and Policy, Icahn School of Medicine at Mount Sinai, New York, New York
\end{abstract}

\begin{abstract}
Keywords

- obstetrics

- COVID-19

- SARS-CoV-2

- protocols

- labor and delivery

- antepartum fetal surveillance

Recently, a novel coronavirus, precisely severe acute respiratory syndrome-coronavirus-2 (SARS-CoV-2), that causes the disease novel coronavirus disease 2019 (COVID-19) has been declared a worldwide pandemic. Over a million cases have been confirmed in the United States. As of May 5, 2020, New York State has had over 300,000 cases and 24,000 deaths with more than half of the cases and deaths occurring in New York City (NYC). Little is known, however, of how this virus impacts pregnancy. Given this lack of data and the risk for severe disease in this relatively immunocompromised population, further understanding of the obstetrical management of COVID-19, as well as hospital level preparation for its control, is crucial. Guidance has come from expert opinion, professional societies and public health agencies, but to date, there is no report on how obstetrical practices have adapted these recommendations to their local situations. We therefore developed an internet-based survey to elucidate the practices put into place to guide the care of obstetrical patients during the COVID-19 pandemic. We surveyed obstetrical leaders in four academic medical centers in NYC who were implementing and testing protocols at the height of the pandemic. We found that all sites made changes to their practices, and that there appeared to be agreement with screening and testing for COVID-19, as well as labor and delivery protocols, for SARS-CoV-2positive patients. We found less consensus with respect to inpatient antepartum fetal
\end{abstract}

\section{Key Points}

- Practices changed to accommodate public health needs.

- Most practices are screened for novel COVID-19 on admission.

- Fetal testing in COVID-19 patients varied.

received

May 15, 2020

accepted

May 16, 2020

published online

June 9,2020
Copyright $\odot 2020$ by Thieme Medical Publishers, Inc., 333 Seventh Avenue, New York, NY 10001, USA. Tel: +1(212) 760-0888.
DOI https://doi.org/

10.1055/s-0040-1713120. ISSN $0735-1631$. 
Recently, a novel coronavirus severe acute respiratory syndrome-coronavirus-2 (SARS-CoV-2) causing the clinical disease novel coronavirus disease 2019 (COVID-19) has been declared a worldwide pandemic, with 3 million cases and 200,000 deaths. $^{1,2}$ The first case reported in the United States occurred in early March. ${ }^{3}$ The United States has had 1,171,510 confirmed cases and 68,279 deaths as of May 5, 2020. ${ }^{4}$ In late March, hospitals in New York City (NYC) were faced with a surge of cases and by May 5, New York State had 313,636 cases and 24,717 deaths with more than half of the cases $(171,723)$ and confirmed deaths $(13,684)$ occurring in NYC. ${ }^{5,6}$ In just 4 weeks, one NYC hospital saw a 40-fold increase in COVID-19 infection among their maternity patients. ${ }^{7}$ As knowledge of the disease evolved, rapid changes in hospital practices were necessary. While the pandemic appears to be plateauing in NYC, the virus is affecting other regions of the United States, and in the coming months, there remains a risk of a second wave in cases.

Recent data from a systematic review of COVID-19 during pregnancy highlighted the outcomes of 51 cases and stresses the importance of additional studies exploring the implications of SARS-CoV-2 infection in pregnancy, as well as the rationaledriving obstetric interventions. ${ }^{8}$ A report from NYC described 43 SARS-CoV-2 infected pregnant women, including seven diagnosed prior to adoption of universal SARS-CoV-2 polymerase chain reaction (PCR) testing. ${ }^{9}$ In this study, $9 \%$ of the women developed severe disease, and almost $5 \%$ had critical disease. This data are consistent with reports from Wuhan where there was an $8 \%$ risk of severe disease in pregnant women. ${ }^{10}$ Given the lack of data, the relatively immunocompromised state of pregnancy, and the potential for severe disease, further understanding of COVID-19 clinical management, as well as hospital level preparation for its control are crucial.

In the United States, both expert opinion and advice from professional societies have guided the clinical management of COVID-19. ${ }^{11-13}$ Guidance on cesarean delivery, prevention of postpartum hemorrhage, and collection of biospecimens have come from obstetrical centers with experience in prior coronavirus outbreaks. ${ }^{14}$ An overview of COVID-19 in pregnant women proposed a framework for the unique complexities and logistics in managing this disease in obstetrics. ${ }^{15}$ Finally, public health agencies have provided guidance on how to modify practices during this pandemic. ${ }^{16}$ To date, the literature is limited on how hospitals are providing obstetric care and have responded to COVID-19. As the threat of the disease remains, hospitals, practitioners, and health systems may benefit from the real-world information gained by centers that have been at the forefront of the pandemic.

Herein, we report a survey of members of the NYC Maternal-Fetal Medicine (MFM) Research Consortium across four health systems during the peak of the pandemic. The survey aims to elucidate the practices put into place to guide patient care after four weeks managing SARS-CoV-2 infections in obstetrical patients. Given the dynamic nature of this disease, practices changed quickly as new issues arose, and our goal was to report our experience to other obstetrical providers, hospitals, and leaders as they formulate their own local-practices in managing the pandemic.

\section{Materials and Methods}

This was an internet-based survey conducted with four sites participating in the NYC MFM Research Consortium during the SARS-CoV-2 pandemic. The NYC MFM Research Consortium was formed in 2017 and in March to April 2020, decided to focus solely on COVID-19-related research. We developed a survey that focused on practices and procedures around the following domains: (1) screening for COVID-19 in patients presenting to labor and delivery; (2) management of SARSCoV-2 infected and noninfected patients undergoing labor and delivery; (3) management of SARS-CoV-2 PCR positive and negative antepartum inpatients; (4) management of neonates of SARS-CoV-2-positive and -negative patients; (5) antepartum fetal surveillance for COVID-19 inpatients; and (6) obstetrical interventions in the COVID-19 patient. Given the nature of the pandemic and our desire to conduct the survey in realtime, we did not perform pretesting for survey validation.

Surveys were administered via Redcap. ${ }^{17}$ Participants were either MFM specialists, or obstetrical service or labor and delivery directors, and received no incentives. Because we wanted to capture practice patterns during the height of the pandemic, the survey time spanned from April 14, 2020 through April 17, 2020. A member of each Consortium site completed 118 questions over six survey pages/screens. Redcap allowed us to check for survey completeness, and respondents were able to review and correct their answers as needed.

Given the small number of centers involved, we did not weight the responses, and did not adjust for nonrepresentative samples. We did not plan to perform a statistical analysis and limited this manuscript to the survey responses. The Institutional Review Board of Mount Sinai Health System approved this survey.

\section{Results}

Four centers agreed to participate in the survey, and all participants completed the survey in its entirety. - Table 1 presents the characteristics of the hospitals surveyed. All the hospitals are tertiary referral centers located in different regions in NYC, representing a diverse patient population. Site 1 included only one hospital within its health system, while the other three sites included two hospitals within their system. In two of the sites, the majority of patients were non-Hispanic Whites, while in the other two, the majority were Hispanic. During the study, the prevalence of SARSCoV-2 infected women presenting to labor and delivery ranged from 8 to $46 \%$.

\section{Personal Protective Equipment}

All sites reported having adequate personal protective equipment (PPE) available, and required staff to wear surgical masks at all times. In SARS-CoV-2 PCR negative or persons under investigation (PUI), most sited limited N95 (N95) respirator use to procedures with a risk of intubation and during the second stage of labor. Only one center required full PPE for care of a PUI or SARS-CoV-2 PCR negative patient. All sites screened patients for COVID-19 using clinical history 


\begin{tabular}{|c|c|c|c|c|}
\hline Hospital & Site 1 & Site 2 & Site 3 & Site 4 \\
\hline Number of total deliveries in 2019 & 4,600 & 5,210 & 13,597 & 10,678 \\
\hline \multirow[t]{4}{*}{ Race/ethnicity distribution ${ }^{\mathrm{a}}(\%)$} & $\begin{array}{l}\text { Non-Hispanic } \\
\text { White } 36\end{array}$ & $\begin{array}{l}\text { Non-Hispanic } \\
\text { White } 6\end{array}$ & $\begin{array}{l}\text { Non-Hispanic } \\
\text { White } 60\end{array}$ & $\begin{array}{l}\text { Non-Hispanic } \\
\text { White } 52\end{array}$ \\
\hline & $\begin{array}{l}\text { Non-Hispanic } \\
\text { Black } 16\end{array}$ & $\begin{array}{l}\text { Non-Hispanic } \\
\text { Black } 29\end{array}$ & $\begin{array}{l}\text { Non-Hispanic } \\
\text { Black } 10\end{array}$ & $\begin{array}{l}\text { Non-Hispanic } \\
\text { Black } 7\end{array}$ \\
\hline & Hispanic 54 & Hispanic 43 & Hispanic 18 & Hispanic 18 \\
\hline & Asian 5 & Asian 4 & Asian 10 & Asian 21 \\
\hline $\begin{array}{l}\text { Number of SARS-CoV-2 }(+) \\
\text { deliveries that occurred } \\
\text { during survey time }\end{array}$ & 32 & 40 & 67 & 30 \\
\hline
\end{tabular}

${ }^{\mathrm{a} S i t e s}$ provided estimates of race/ethnicity.

and temperature on presentation to labor and delivery. Most sites performed universal nasopharyngeal PCR for SARSCoV-2 on admission, or 24 to 48 hours prior to a scheduled admission, although, since the time of survey completion, all four sites adopted this procedure. The majority of sites also screened the patient's support person using clinical symptoms and temperature checks during admission. One center performed SARS-CoV-2 PCR testing for all support persons either on admission to labor and delivery (L\&D), or 24 to 48 hours prior to a scheduled admission.

\section{Practices and Procedures for SARS-CoV-2 PCR Negative or Persons under Investigation}

Most sites made changes to their procedures and protocols forSARS-CoV-2 negative and PUI (-Table 2). Early in the pandemic, centers prohibited a support person. This policy was changed, and by the time of the survey, all sites, allowed only one support person. All sites recommended early epidural placement to reduce chance of general anesthesia in the case of emergency cesarean. Nitrous oxide analgesia was suspended in the one site where it was available. Most sites continued to perform delayed cord clamping. No sites used oxygen for nonreassuring fetal heart rate tracing (NRFHT), and most sites continued to use carboprost for the management of postpartum hemorrhage (PPH). All centers also continued to use nonsteroidal anti-inflammatory drugs (NSAIDS). During the postpartum period, most centers cohorted patients based on SARS-CoV-2 status. All centers allowed rooming in of newborns. All sites had a nursery available for newborns of SARSCoV-2-negative mothers. No sites tested newborns and the majority of sites continued to perform circumcisions. All sites continued to make lactation consultation available. All sites encouraged early discharge if appropriate (at 24 hours for vaginal deliveries or 48 hours for cesarean deliveries) and performed a phone follow-up shortly after discharge.

\section{Practices and Procedures forSARS-CoV-2 PCR Positive Patients}

In SARS-CoV-2-positive pregnancies, most sites changed policies to attempt to limit virus exposure ( - Table 3 ).

\begin{tabular}{|llllr|}
\hline \multicolumn{2}{|l|}{ Table 2 Labor and delivery/postpartum practices and procedures for SARS-CoV-2 negative or person under investigation } \\
\hline Practices and procedures & Site 1 & Site 2 & Site 3 & Site 4 \\
Only one support person allowed & Yes & Yes & Yes & Yes \\
Early epidural recommended & Yes & Yes & Yes & Yes \\
Nitrous oxide suspended & N/A & N/A & Yes & N/A \\
All cesareans performed in an AlIR OR & No & No & No & No \\
O2 for NRFHRT & No & No & No & No \\
carboprost for PPH & Yes & Yes & Yes & Yes \\
NSAID used & Yes & Yes & Yes & Yes \\
Cohorting patients based on SARS-CoV-2 status & Yes & No & Yes & $4 / 4$ \\
Offering early discharge to all patients & Yes & No & Yes & Yes \\
Phone follow-up for patients discharged early & Yes & Yes & Yes & Yes \\
\hline
\end{tabular}

Abbreviations: AIIR, airborne infection isolation room (also known as negative pressure room); N/A, not available; NRFHRT, nonreassuring fetal heart rate tracing; NSAID, nonsteroidal anti-inflammatory drug; OR, operating room; PPH, postpartum hemorrhage; SARS-CoV-2, severe acute respiratory syndrome-coronavirus-2.

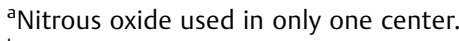

bonly used in known negatives. 


\begin{tabular}{|c|c|c|c|c|c|}
\hline Practices and procedures & Site 1 & Site 2 & Site 3 & Site 4 & All \\
\hline If available, vaginal delivery performed in an AIIR room on L\&D & Yes & Yes & Yes & Yes & $4 / 4$ \\
\hline Vaginal delivery performed in an AIIR room in the main OR & No & No & No & No & $0 / 4$ \\
\hline Cesarean performed in standard OR on L\&D & Yes & Yes & Yes & $Y_{e s}{ }^{\mathrm{a}}$ & $3 / 4$ \\
\hline Cesarean performed in an AIIR room on $L \& D$ & No & No & No & $\mathrm{No}^{\mathrm{a}}$ & $3 / 3$ \\
\hline Cesarean performed in standard room in the main OR & No & Yes & No & No & $1 / 4$ \\
\hline Cesarean performed in an AIIR room in the main OR & No & No & No & Yes & $1 / 4$ \\
\hline Critically ill delivered in an AlIR in main OR & No & No & Yes & Yes & $2 / 4$ \\
\hline Cord blood banking collection suspended & No & $\mathrm{N} / \mathrm{A}$ & Yes & Yes & $2 / 3$ \\
\hline Labor nurse in and out of room & Yes & Yes & Yes & Yes & $4 / 4$ \\
\hline IV pumps and tubing outside of room & Yes & No & Yes & No & $2 / 4$ \\
\hline Modified PPE procedure to accommodate urgent cesarean & Yes $^{\mathrm{b}}$ & Yes & No & No & $1 / 4$ \\
\hline carboprost used & No & Yes & No & No & $1 / 4$ \\
\hline NSAID used & Yes $^{c}$ & Yes & Yes & Yes & $3 / 4$ \\
\hline Thromboprophylaxis for 2 weeks postpartum ${ }^{e}$ & Yes & Yes $^{\mathrm{d}}$ & Yes & No & $3 / 4$ \\
\hline
\end{tabular}

Abbreviations: AlIR, airborne infection isolation room (also known as negative pressure room); IV, intravenous; L\&D, labor and delivery; N/A, not applicable; NSAID, nonsteroidal anti-inflammatory drug; OR, operating room; PCR, polymerase chain reaction; PPE, personal protective equipment; SARS-CoV-2, severe acute respiratory syndrome-coronavirus-2; VTE, venous thromboembolism.

${ }^{a} Y e s$, only if negative pressure in main OR unavailable, then perform with HEPA filter.

${ }^{\mathrm{b}}$ Case by case

'Use caution in ordering NSAIDs for symptomatic postpartum patients with novel coronavirus disease 2019 (COVID-19) infection and/or asymptomatic COVID-19 with chronic kidney disease.

dSometimes

ésites used either heparin or enoxaparin for thromboprophylaxis.

When available, all sites performed vaginal deliveries in an airborne infection isolation room (AIIR, also known as negative pressure room). Most sites performed cesarean deliveries in a standard operating room (OR; without negative pressure) on L\&D. One site performed all SARS-CoV-2-positive cesareans in a negative pressure room in the main OR. Most centers suspended cord blood banking. Most centers protected nursing staff by limiting direct patient contact and some centers placed intravenous (IV) pumps and tubing outside of the room. Most centers discharged patients with a 2-week course of prophylactic heparin or enoxaparin. Unlike for SARS-CoV-2 negative patients, the majority of sites did not use carboprost for the management of PPH in SARS-CoV-2-positive patients. Starting at 23 weeks, most centers would give corticosteroids for fetal lung maturity and magnesium sulfate for SARS-CoV-2 patient with signs or symptoms of preterm labor or preeclampsia. The majority of centers continued rooming in of neonates. Universally, newborn testing occurred at 24 hours; however, one center repeated testing at 48 hours if the initial test was negative. Most centers cohorted newborns of SARS-CoV-2-positive mothers in the neonatal intensive care unit (NICU) or the newborn nursery. No site routinely isolated newborns in the hospital for 14 days.

\section{Antepartum Fetal Surveillance in SARS-CoV-2 PCR- Positive Hospitalized Patients}

There was less consensus on antepartum fetal surveillance in COVID-19 patients. For example, there was variability in the level of fetal testing for a COVID-19, non-ICU patient, with a viable fetus. One site would do no testing until 34 weeks, and another would start at 24 weeks ( - Table 4 ). One site would not start testing until 26 weeks. When patients were admitted to the ICU, but not intubated, half of the respondents performed daily non-stress tests (NSTs) while half performed only daily fetal heart (FH) tone checks. One site had an obstetrical ICU and performed continuous monitoring for all ICU patients, regardless of their clinical status. For ICU patients who were intubated with viable fetuses, half of the sites monitored by FHs check, while the other half performed an NST. In a patient with a fetus beyond 24 weeks of gestation that was decompensating, half of the centers would perform a FH check, while one center would do an NST, and one would not monitor the fetus. For half of the sites, after 34 weeks, the risks of continued expectant management of a patient with COVID-19 seemed to outweigh the risks of prematurity, and these centers would forgo testing and recommend delivery.

\section{Obstetrical Interventions for SARS-CoV-2-Positive Patients}

In a critically ill COVID-19 patient, all centers would perform an emergent cesarean for worsening maternal status starting at 24 weeks. For worsening fetal status, the majority would perform an emergent cesarean starting at 28 weeks. There was less consensus on emergent delivery for fetal indications at less than 28 weeks, as only half of the sites would intervene, and only under certain circumstances after shared decision making with the patient. For an unstable COVID-19 in the ICU, most 


\begin{tabular}{|c|c|c|c|c|}
\hline Clinical scenario & Site 1 & Site 2 & Site 3 & Site 4 \\
\hline $\begin{array}{l}23-33^{6} \text { weeks of GA: } \\
\text { mother stable }\end{array}$ & $N / A$ & $\begin{array}{l}<26 \text { FH checks, } \\
>26 \text { weeks daily NST }\end{array}$ & $\begin{array}{l}\text { None until } \\
34 \text { weeks }\end{array}$ & $\begin{array}{l}\text { Daily NST starting } \\
\text { at } 24 \text { weeks }\end{array}$ \\
\hline $\begin{array}{l}23-33^{6} \text { weeks of GA: } \\
\text { mother in the ICU, } \\
\text { not intubated }\end{array}$ & Continuous & $\begin{array}{l}<26 \text { weeks FH checks, } \\
>26 \text { weeks daily NST }\end{array}$ & $\begin{array}{l}\text { None until } \\
34 \text { weeks }\end{array}$ & $\begin{array}{l}\text { Daily NST starting } \\
\text { at } 24 \text { weeks }\end{array}$ \\
\hline $\begin{array}{l}23-33^{6} \text { weeks of GA: } \\
\text { mother in the ICU, } \\
\text { intubated }\end{array}$ & Continuous & $\begin{array}{l}<28 \text { weeks FH checks, } \\
>28 \text { weeks daily NST }\end{array}$ & $\begin{array}{l}\text { None until } \\
34 \text { weeks }\end{array}$ & $\begin{array}{l}\text { Daily NST starting } \\
\text { at } 24 \text { weeks. }\end{array}$ \\
\hline $\begin{array}{l}23-33^{6} \text { weeks of GA: } \\
\text { mother in the ICU, } \\
\text { decompensating }\end{array}$ & Continuous & $\begin{array}{l}<28 \text { weeks FH checks, } \\
>28 \text { weeks daily NST }\end{array}$ & $\begin{array}{l}\text { None until } \\
34 \text { weeks }\end{array}$ & $\begin{array}{l}\text { Daily NST starting } \\
\text { at } 28 \text { weeks }\end{array}$ \\
\hline $\begin{array}{l}\text { 34+ weeks of GA: } \\
\text { mother in the ICU, } \\
\text { not intubated }\end{array}$ & Continuous & Deliver & Deliver & Deliver \\
\hline $\begin{array}{l}34+\text { weeks of GA: } \\
\text { mother in the ICU, } \\
\text { intubated }\end{array}$ & Continuous & Deliver & Deliver & Deliver \\
\hline $\begin{array}{l}34+\text { weeks of GA: } \\
\text { mother in the ICU, } \\
\text { decompensating }\end{array}$ & Continuous & Deliver & Deliver & Deliver \\
\hline
\end{tabular}

Abbreviations: COVID-19, novel coronavirus disease 2019; FH, fetal heart; GA, gestational age; ICU, intensive care unit; NST, non-stress test; N/A, not answered.

centers would deliver the patient, if possible, in an AIIR room in the main OR. Half of the centers would deliver a patient with COVID-19 symptoms who is otherwise stable and with reassuring fetal status between 34 to 36 weeks. Seventy five percent of the sites would deliver a stable COVID-19 patient with reassuring fetal status between 37 to 39 weeks. Finally, most sites were using novel COVID-19 treatments and recommending thromboprophylaxis (-Table 5).

\section{Discussion}

Here we report on the obstetrical practices and protocols from four academic medical centers in NYC at the height of the COVID-19 pandemic. All sites made changes to their practices that were consistent with public health recommendations and practical for local circumstances. There appeared to be agreement with screening and testing for
COVID-19, as well as L\&D protocols for SARS-CoV-2-positive patients, particularly involving use of PPE, and medication use such as corticosteroids, magnesium sulfate, carboprost, and thromboprophylaxis, as well as procedures for newborn testing. We found less consensus with respect to inpatient antepartum fetal surveillance.

While we were unable to document specific practice changes from weeks 1 to 4, there was evidence of independent changes in practice by the time of the survey and after. For example, very early in the pandemic, hospitals prohibited support persons from accompanying laboring patients. This policy changed by the time the survey was performed. Additionally, at the time of the survey, only half the centers were doing universal testing. As of this writing, all centers have adopted this policy. Knowledge of SARS-CoV-2 status may affect downstream decisions, and identifying infected patients allows for appropriate cohorting and protection of staff. If a center elects a strategy of selective

\begin{tabular}{|c|c|c|c|c|c|}
\hline & -Table 1 & -Table 2 & -Table 3 & -Table 4 & All \\
\hline Azithromycin & Yes & No & Yes & Yes & $3 / 4$ \\
\hline Hydroxychloroquine & Yes & Yes & Yes & Yes & $4 / 4$ \\
\hline Remdesivir & Yes & No & Yes & Yes & $3 / 4$ \\
\hline Monoclonal antibody & Yes & No & Yes & Yes & $3 / 4$ \\
\hline Plasmapheresis & No & No & Yes & Yes & $2 / 4$ \\
\hline Outpatient thromboprophylaxis ${ }^{\mathrm{a}}$ SARS-CoV-2-positive patients & Yes & Yes & Yes & No & $3 / 4$ \\
\hline Prone ventilation & No & Yes & No & Yes & $2 / 4$ \\
\hline
\end{tabular}

Abbreviations: COVID-19, novel coronavirus disease 2019; SARS-CoV-2, severe acute respiratory syndrome-coronavirus-2.

${ }^{a}$ Sites used either heparin or enoxaparin for thromboprophylaxis. 
testing, a broader list of inclusion criteria including common obstetrical complications that may be confused with COVID-19 (i.e., preeclampsia and chorioamnionitis) is advised. ${ }^{18,19}$ One site with a high prevalence of COVID-19 found testing of support persons valuable because it guided which visitors could stay to support the patient, while limiting risk for other patients and staff. While there was less consensus with respect to inpatient antepartum fetal surveillance, all sites reported establishing a clear policy that delineated at what gestational age to start and what patients would be candidates for testing. This is useful when counseling both patients and other members of the health care team.

\section{Strengths and Limitations}

Our study has several strengths. We surveyed leaders of obstetrical units in NYC immersed in the development and testing of new protocols in an early epicenter of the pandemic. We document the practices put into place after 4 weeks of planning and caring for pregnant COVID-19 patients. The sites surveyed also cared for a large diverse patient population. Finally, we were able to demonstrate how sites independently arrived at similar conclusions in certain areas while differing practices in others. We also acknowledge several limitations that could introduce bias. This survey included only large, urban centers with access to robust resources. One site had an obstetrical ICU, an uncommon practice model nationally. This may make some of the protocols less generalizable to smaller centers. Additionally, resources varied at different sites and so consensus on areas such as negative pressure operating rooms or delivery rooms could not be obtained.

\section{Conclusion}

Several expert commentaries have provided guidance on PPE, procedures during L\&D, and management of common obstetrical issues during the pandemic. ${ }^{20-22}$ A recent Cochrane review aimed at compiling international guidelines related to COVID-19 during pregnancy. ${ }^{23}$ This group found international consensus in a few important areas, including which pregnant women should wear a mask or respirator, and how centers were managing support persons in asymptomatic women. ${ }^{23}$ Less consensus was noted with respect to antepartum fetal surveillance in COVID-19 patients, or whether COVID-19 was a contraindication to antenatal steroids for fetal lung maturity. To our knowledge, this is the first survey to document obstetrical protocols and procedures put into place at multiple centers within an urban setting during the COVID19 pandemic, and that specifically surveyed policies on inpatient antepartum fetal surveillance and corticosteroids for fetal lung maturity. While we are not able to say whether the protocols mentioned here represent "the best practices," they do represent practices that were developed over time to best care for patients, and at the same time to protect health care workers. Ultimately, our hope is that sharing policies such as SARS-CoV-2 universal testing on patients in labor or scheduled for delivery, cohorting patients, antepartum fetal surveil- lance, and obstetrical interventions will help simplify and direct care for a COVID-19 obstetrical patients.

Conflict of Interest

None declared.

\section{References}

1 World health Organization. Coronavirus disease 2019 (COVID19) situation report-75. Available at: https://www.who.int/docs/ default-source/coronaviruse/situation-reports/20200404-sitrep75-covid-19.pdf?sfvrsn=99251b2b_4. Accessed May 20, 2020

2 Dong E, Du H, Gardner L. An interactive web-based dashboard to track COVID-19 in real time. Lancet Infect Dis 2020;20(05):533-534

3 Holshue ML, DeBolt C, Lindquist S, et al; Washington State 2019nCoV Case Investigation Team. First case of 2019 novel coronavirus in the United States. N Engl J Med 2020;382(10):929-936

4 Centers for Disease and Control. Cases in the U.S. Available at: https://www.cdc.gov/coronavirus/2019-ncov/cases-updates/ cases-in-us.html. Accessed April 24, 2020

5 New York City Department of Health and Mental Hygiene COVID19. COVID-19: Data-Summary. Available at: https://www1.nyc. gov/site/doh/covid/covid-19-data.page. Accessed April 16, 2020

6 New York State Department of Health COVID-19 Information Center. Available at: https://coronavirus.health.ny.gov/home. Accessed May 5, 2020

7 Tekbali A, Grunebaum A, Saraya A, McCullough L, Bornstein E, Chervenak FA. Pregnant versus non-pregnant SARS-CoV-2 and COVID-19 hospital admissions: the first 4 weeks in New York. Am J Obstet Gynecol 2020 (e-pub ahead of print). Doi: 10.1016/j. ajog.2020.04.012

8 Della Gatta ANRR, Pilu G. Simonazzi. COVID19 during pregnancy: a systematic review of reported cases. Am J Obstet Gynecol 2020 (e-pub ahead of print). Doi:

9 Breslin N, Baptiste C, Gyamfi-Bannerman C, et al. COVID-19 infection among asymptomatic and symptomatic pregnant women: two weeks of confirmed presentations to an affiliated pair of New York City hospitals. Am J Obstet Gynecol MFM 2020 (e-pub ahead of print). Doi: 10.1016/j.ajogmf.2020.100118

10 Chen L, Li Q Zheng D, et al. clinical characteristics of pregnant women with COVID-19 in Wuhan, China. N Engl J Med 2020

11 American College of Obstetricians and Gynecologist. Novel coronavirus 2019 (COVID-19). Available at: https://www.acog. org/clinical/clinical-guidance/practice-advisory/articles/2020/ 03/novel-coronavirus-2019. Accessed April 162020

12 The Society for Maternal-Fetal Medicine (SMFM). Coronavirus (COVID-19) and pregnancy: what maternal-fetal medicine subspecialists need to know. Available at: https://s3.amazonaws.com/cdn. smfm.org/media/2317/COVID19-What_MFMs_need_to_know_revision_4-11-20_(final)_PDF.pdf. Accessed April 16, 2020

13 Professional Committee of Maternal and Fetal Physicians of the Obstetrics and Gynecology Branch of the Chinese Medical Doctor Association tOGotOaGBotC. Expert advice on new coronavirus infections during pregnancy and puerperium. Chinese Journal of Perinatal Medicine. 2020;23(02):73-79

14 Qi H, Luo X, Zheng Y, et al. Safe delivery for COVID-19 infected pregnancies. BJOG 2020 (e-pub ahead of print). Doi: 10.1111/14710528.16231

15 Dashraath P, Wong JLJ, Lim MXK, et al. Coronavirus disease 2019 (COVID-19) pandemic and pregnancy. Am J Obstet Gynecol 2020: S0002-9378(20)30343-4

16 Centers for Disease Control and Prevention. Considerations for Inpatient Obstetric Healthcare Settings. Available at: https:// www.cdc.gov/coronavirus/2019-ncov/hcp/inpatient-obstetrichealthcare-guidance.html. Accessed April 17, 2020

17 Harris PA, Taylor R, Thielke R, Payne J, Gonzalez N, Conde JG. Research electronic data capture (REDCap)-a metadata-driven methodology 
and workflow process for providing translational research informatics support. J Biomed Inform 2009;42(02):377-381

18 Guan WJ, Ni ZY, Hu Y, et al; China Medical Treatment Expert Group for Covid-19. Clinical characteristics of coronavirus disease 2019 in China. N Engl J Med 2020;382(18):1708-1720

19 Zheng Z, Peng F, Xu B, et al. Risk factors of critical \& mortal COVID19 cases: A systematic literature review and meta-analysis. J Infect 2020:S0163-4453(20)30234-6

20 Jamieson DJ, Steinberg JP, Martinello RA, Perl TM, Rasmussen SA. Obstetricians on the coronavirus disease 2019 (COVID-19) front lines and the confusing world of personal protective equipment. Obstet Gynecol 2020
21 Rasmussen SA, Jamieson DJ. Coronavirus disease 2019 (COVID$19)$ and pregnancy: responding to a rapidly evolving situation. Obstet Gynecol 2020;135(05):999-1002

22 Stephens AJ, Barton JR, Bentum NA, Blackwell SC, Sibai BM. General guidelines in the management of an obstetrical patient on the labor and delivery unit during the COVID-19 pandemic. Am J Perinatol 2020

23 COVID-19 review of national clinical practice guidelines for key questions relating to the care of pregnant women and their babies. Available at: https://pregnancy.cochrane.org/news/covid-19-review-national-clinical-practice-guidelines-key-questions-relating-care-pregnant. Accessed May 11, 2020 